\title{
TOPIRAMATE THERAPY AND COGNITIVE DYSFUNCTION
}

The effects of topiramate (TPA) adjunctive therapy on cognition in 22 consecutive patients with intractable epilepsy were studied at the Montreal Neurological Hospital, Quebec, Canada. Performance on neuropsychological tests administered on and subsequently off TPM was analyzed. In a second study at the Minnesota Epilepsy Group, St Paul, MN, 16 patients were tested first off, then on TPM. In the Quebec study, significant improvements were observed after discontinuing TPM on 13 measures of verbal and nonverbal fluency and some perceptual tasks. In the Minnesota study, performance was impaired on all tests of cognition after TPM was begun, and especially for tests of fluency, sustained concentration, and visual motor processing speed. (Lee S, Sziklas V, Andermann F, et al. Epilepsia March 2003;44:339-347). (Respond: Dr Suzee Lee, McGill University, Montreal Neurological Hospital, Monytreal, Quebec, Canada).

COMMENT. Topiramate is associated with impairments of fluency, attention/concentration, processing speed, language skills, and perception. Working memory but not retention is affected. The TPM-induced cognitive dysfunction is independent of the order of testing, first on or off medication.

Topiramate-asociated word-finding difficulties are reported from the Institute of Neurology, University College, Queen Square, London (Mula M, Trimble MR, Thompson P, Sander J. Neurology April 8, 2003;60:1104-1107). Word-finding difficulties developed in $31(7.2 \%)$ patients with epilepsy during treatment with TPM. Patients with simple partial seizures and a left temporal EEG epileptic focus were especially at risk.

\section{MACROCEPHALY AND RISK OF SEIZURES}

The epidemiology, perinatal risk factors, and major neurologic comorbidity of 42 children with hydrocephalus were studied in a community-based clinic for children with neurodevelopmental disabilities at the Institute for Child Development, Tel Aviv, Israel. Of 4,309 children examined, $62(1.4 \%)$ had macrocephaly $\left(\mathrm{HC}>98^{\text {th }}\right.$ percentile), of whom $42(1 \%)$ had macrocephaly not associated with hydrocephalus. Of the 42,3 had familial macrocephaly and 15 had comorbid diagnoses: generalized macrosomia in 6, of whom 3 had cerebral gigantism (Soto's syndrome), migration defects (2), holoprosencephaly (1), neurofibromatosis type 1 (1), achondroplasia (2), myotonic dystrophy (1), and other (2). In comparison with normocephalic disabled controls, children with macrocephaly without hydrocephalus had an increased incidence of neonatal respiratory distress (9.5\% vs $3.6 \%$, $\mathrm{p}=0.042$ ); other perinatal complications were not significantly different in the 2 groups. In the 42 patients with macrocephaly without hydrocephalus the risk of developing epilepsy or febrile seizures was significantly increased compared with controls $(24 \%$ and $10 \%$ $[\mathrm{p}<0.001$ and $\mathrm{p}=0.026]$ respectively). Macrocephaly was a significant risk factor for febrile seizures (odds ratio $=3.1, p<0.001$ ) and epilepsy (odds ratio $=7.7, p<0.001$ ), but not for mental retardation (odds ratio $=1.1, \mathrm{p}=0.78$ ) or cerebral palsy (odds ratio $=1.3, \mathrm{p}=0.67$ ). (Nevo Y, Kramer U, Shinnar S, et al. Macrocephaly in children with developmental disabilities. Pediatr Neurol Nov 2002;27:363-368). (Respond: Dr Nevo, The Institute for Child Development, 14 Balfour St, Tel Aviv, 65211, Israel). 
COMMENT. Macrocephaly in children with diabilities, even when not associated with hydrocephalus, is associated with an increased risk of seizures. In addition to hydrocephalus, causes for macrocephaly include true megalencephaly, subdural effusion, pseudotumor cerebri, and familial variant. Megalencephaly may be a hamatomatous malformation, or is associated with metabolic CNS diseases (leukodystrophies and lipidoses), neurocutaneous syndromes, Sotos' syndrome, and achondroplasia.

Lorber (1981) reported on a series of 510 children hospitalized because of macrocephaly. Of these, $75 \%$ had increased intracranial pressure, and $20 \%$ had primary megalencephaly. In children with megalencephaly, the male to female ratio was 4 to 1 , familial incidence was $50 \%$, and intellectual retardation and abnormal neurologic findings occurred in 13\%. Abnormal head growth occurred mainly in the first 4 months.

Macrocephaly and epidural mass with acute lymphoblastic leukemia are reported in a 2-year-old female. Chemotherapy led to remission of ALL and MRI resolution of the mass. Macrocephaly may be a rare and early manifestation of leukemia. (Jaing T-H, Hung P-C, Hung I-J, et al. Pediatr Neurol 202;27:401-403).

\section{HEADACHE DISORDERS}

\section{BEHAVIORAL TREATMENT FOR TENSION-TYPE HEADACHE}

The benefits of brief neurologist-administered behavioral treatment of pediatric episodic tension-type headache were determined at the University of West Florida, Pensacola, FL. Thirty seven children, 26 females and 11 males, mean age 12.3 years (range 9-16 years), with a minimum of one headache per week, were included. Patients kept daily headache diaries for monthly periods, before treatment and at follow-up at 1, 3, 6, and 12 months. Days of headache activity, analgesic tablet counts, and responder rate were the main outcome measures. Patients were seen for a maximum of 30 minutes in groups of 3 to 5 , once per week for 8 weeks. Sessions consisted of a review of the prior week, analysis of and coping with headache-eliciting situations, and practice of progressive muscle relaxation training with 8 muscle groups. The first session was tape recorded to guide home practice, and the tape was used once per day during treatment and twice per week thereafter. The treatment sessions were designed by a behavioral psychologist. Improvements, except for the use of analgesics, were significant and persisted through 1 year follow-up. Analgesic tablet consumption was reduced by more than $50 \%$, but the reduction was not significant. (Andrasik F, Grazzi L, Usai S, et al. Brief neurologist-administered behavioral treatment of pediatric episodic tension-type headache. Neurology 8 April 2003;60:1215-1216). (Reprints: Dr F Andrasik, Institute for Human and Machine Cognition, University of West Florida, 40 S Alcaniz St, Pensacola, FL 32501).

COMMENT. Neurologist administered, group treatment sessions involving muscle relaxation exercises and discussion of headache-eliciting situations may offer an alternative to supplemental medication in children with tension-type headaches. Since the behavioral psychologist designed and is trained to conduct group therapy, it may be more appropriate and cost-effective to refer the patient for this form of management. 